\title{
A Sleep-Awakening Communication Technology for Transmission Line Network
}

\author{
Jiang Zhu \\ State Grid Electric Power Research Institute \\ Nanjing, Jiangsu, 210003, China
}

\begin{abstract}
Equipment on the transmission line generally works in the absence of the utility of the wild environment. When using solar, wind and other power supply, communications equipment will be faced with the problem of power consumption. Communication technology proposed in this paper, aimed at solving low power communication technology, especially the function of transmission lines under the special environment with communication of intelligent energy saving problem of the equipment.
\end{abstract}

Keywords-transmission line network; low power; sleepawakening

\section{INTRODUCTION}

Along with the development of information technology such as condition monitoring [1], the functional sex of the transmission line will be the deployment of more and more dependent on the communication of the intelligent device. In many cases, it needs to support two or more ways of communication. Equipment on the transmission line, however, usually work in the absence of the utility of the wild environment. In the way when the power is supplied by solar energy, wind energy and so on, the communication function of power consumption problems [2] inevitably happen.

Most of the intelligent devices on the transmission line include embedded processing components and peripheral communication module. At present, the processing ability of the embedded processor and components on the market types is various, and there are a lot of MCU have proprietary mechanism of low power consumption, can through certain MCU selection and system design to satisfy needs. However, a variety of standard protocol communication module power consumption is very high, in the absence of mains power supply, difficult to maintain effective communication for a long time. If changes in the underlying the communication protocol to meet the needs of the power consumption[3-9], is bound to affect the rate, and significantly sacrifice communication mode to the variety of the compatibility, cannot be applied to the deployed environment of communication facilities. How to ensure that meet the needs of the communication situation to reduce the power consumption of the whole device as far as possible, so as to adapt to the environment field, is all sorts of intelligent equipment on the transmission line, a key issue.

In this paper, the transmission line dormancy activation communication technology, low power consumption is based on the demand for communication capacity, establish a set of dormancy activation mechanism, thus use by subdivision of communication resources, reduce the power consumption of the whole system.

\section{COMMUNICATION PROCESS}

Equipment on the transmission line, generally not has been in a state of data communication with other devices, is usually a periodic data exchange or the discovery of emergency data report. Its network generally has two kinds of structures: planar structure and hierarchical structure. In planar structures, all nodes are equal, so it can be called the equality structure. A hierarchical structure, network is divided into clusters of mow. Each cluster is made up of a cluster head and a plurality of cluster members. These cluster heads form a high level of network. In the high level of the network, and can be divided into clusters, again to form a higher level of network, until the most advanced. In the hierarchical structure, the cluster head node is responsible for forwarding the data of the cluster. The cluster head can be specified in advance, and it can be generated automatically by using the algorithm of node. Hierarchical structure of the network can be divided into two kinds of single frequency and multi frequency classification. In a single frequency network, all nodes use the same frequency communication. In order to achieve the communication between the cluster head, the support of the gateway node (the nodes belonging to two clusters) is also supported. In the multi frequency packet network, different levels of communication frequency are used. Lower node communication range is smaller, and higher nodes to cover a larger range. Advanced nodes are in multiple stages, with multiple frequencies, different frequency to achieve different levels of communication. In the two level networks, the cluster head node has two frequencies. Frequency 1 for the communication is between the cluster head and the cluster member. And frequency 2 for the communication is between the cluster head. Each node of the hierarchical network can become a cluster head, so the appropriate cluster head election algorithm, the algorithm should be based on the network topology changes. Flat structure of the network is relatively simple, all nodes in the network is completely equal, in principle, there is no bottleneck, so the more robust. Its disadvantage is that it can be expanded: each node needs to know the route to the other nodes. Maintaining these dynamic changes of routing information requires a large amount of control messages. In the hierarchical structure of the network, the function of the cluster members is relatively simple, without the need to maintain the complex routing information. 
This greatly reduces the number of control information in the network, so it has a good scalability. Because the cluster head node can be elected at any time, the hierarchical structure also has a strong survivability. The drawback of hierarchical structure is that the maintenance of hierarchical structure requires nodes to perform cluster head election algorithm, cluster head node may become the bottleneck of the network. Therefore, when the network size is small, it can be used in a simple planar structure; and when the network size increases, the application of hierarchical structure.

It is equal and equipment on the transmission line, in addition to interact with the backend system, they are also divided into master device and slave device. In this paper, based on the characteristics of this communication, a kind of dormancy mechanism is designed.

\section{SLEEP MECHANISM DESIGN}

The entire device according to the running power consumption can be divided into six working status: deep dormancy and dormancy, hazy, awakening, excited and very excited. Fig 1 depicts the working state of mutual conversion process.

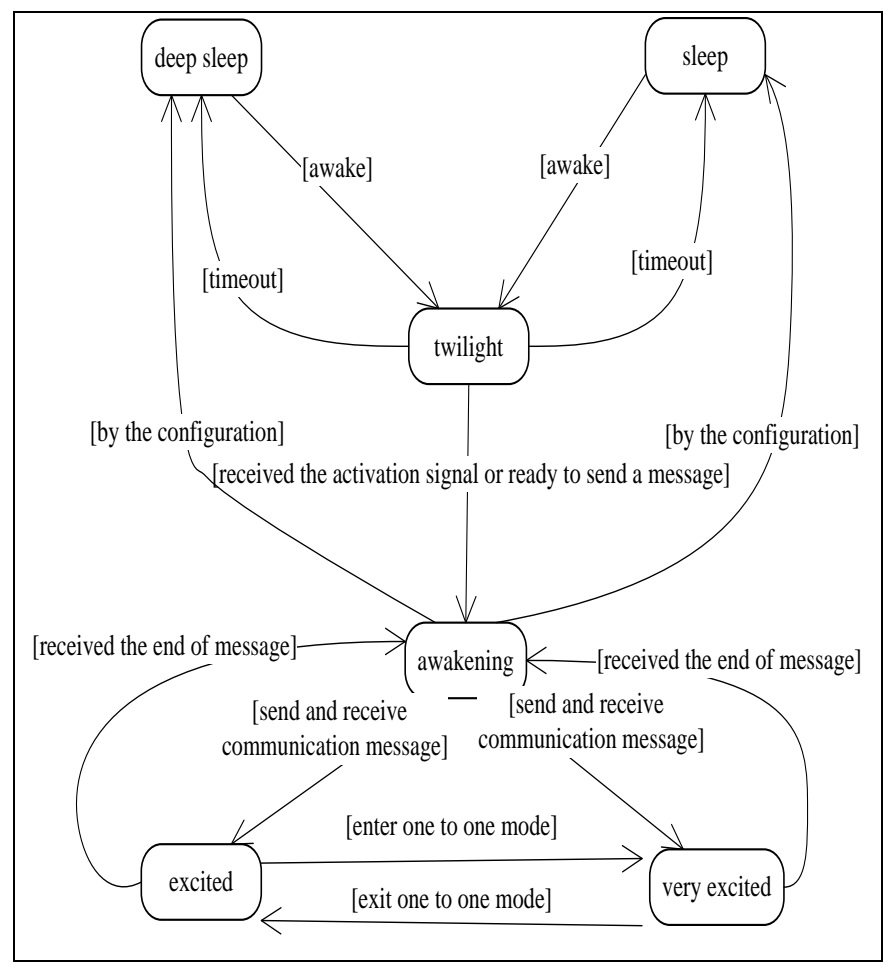

FIGURE I. WORKING STATE TRANSITION DIAGRAM

Deep sleep state: state running the lowest power consumption equipment in this work; don't accept any external signal, all communications devices are shut down, only on the periodic data or data polling to actively, into a twilight state. A long deep sleep wake cycle usually and related to the types of professional data interaction.

Sleep state: Equipment in the work state of running just a little higher than deep dormancy state (far lower than the rest of the work), don't accept any external signal, all communications devices are shut down, only periodic active awakening, into a twilight state. Awakening dormant period is usually short, and has nothing to do with the type of professional data interaction.

Twilight state: Equipment in the working state of running higher than dormancy and deep dormancy (less than the rest of the work). After entering a twilight state, equipment if ready to send a message, will immediately enter the sensei working condition; if equipment is ready to receive a message, it has been in a low power consumption state of communication waiting, until receive activation signals into sensei or wait for a timeout to dormancy and deep dormancy.

Awakening state: In the awakening state, the equipment can normal send and receive all kinds of application layer communication message. At this time, the main equipment can beat polling power saving mode from the device. By a certain time interval between master and slave device information such as transmission pair, command, inform each other. When the main equipment to receive each other's right after the command, enter a state of excitement or extremely excited state, to achieve data interaction between master-slave equipment.

Excited state: When the master-slave equipment of general data exchange between they need to be from the awakening state to enter the state. In the excited state, the power saving mode is used in the master equipment can give priority to polling from the device. Master-slave equipment should send messages each other for many times through the small time interval between them, keep the connection relationship, until both sides mutual information after confirmation has been completed data interaction. They enter the awakening state and then according to the configuration requirements, enter the sleep state or the deep sleep state.

Very excited state: Large amount of data need to transfer between master-slave equipment. When the master-slave equipment need to peer communication (one to one), they need from the awakening state to enter the state.In the very excited state, Master-slave equipment continue to wake up each other and running, until both sides mutual information confirm completed data interaction and parameter configuration. And then they enter the awakening state, in accordance with the requirements of master-slave device configuration, enter the sleep state or deep sleep state.

\section{IMPLEMENTATION WAY}

Obviously, it is not suitable for the design of the hardware and software design of the low power consumption.

From the hardware point of view, to find all possible consumption current loop, and determine what power can be optimized by means of the control software, which is inevitable, and to program compilers provide an all IO port state influence on power relations (usually with simple form explain how the high level and low level how, the suspension will what is enough, do not need to fine to specific numerical. To do this, the basic hardware work will be completed; the rest is the software development staff to play space. And the power 
consumption reduction strategy based on software is the focus of this paper.

When it comes to software power optimization, it is simple and simple, complex and complex. Simple summary of the past is: Application module, functional tasks, task cycle, power consumption, sleep, one vote veto. Not simple enough? And then concentrate is: can sleep on the dormancy, how to sleep. Ha ha...... The estimate is too simple to lose the amount of information,

The implementation of the sleep mechanism need the collaboration of master control module, communication module and power management module. Master control module is responsible for the control of communication module and power management module, and in the deep sleep state and the sleep state close communication module to save power consumption.

In the twilight state, communication module activation of the signal can be transmitted through link layer and network layer by means of high frequency radio. In the awakening state, communication module maintains continuous message interaction under main control module's control. In the excited state and very excited state communication module is operating at full power. Fig 2 depicts the entire unit of function modules.

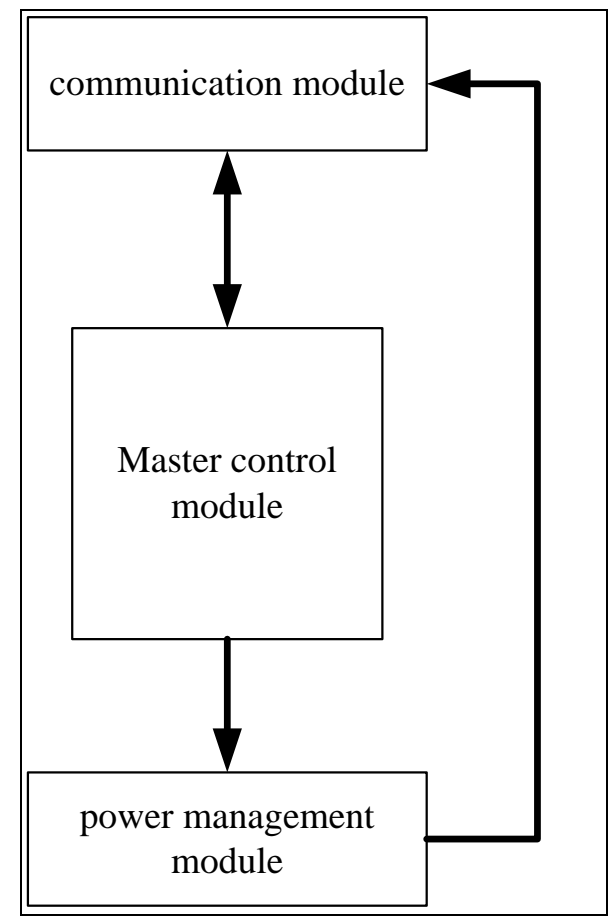

FIGURE II. SCHEMATIC DIAGRAM OF FUNCTION MODULES

A specific application usually consists of many sub functions, sub task. People who understand the architecture of the embedded system is more understandable: an application is implemented by calling a number of services (servie). This service can hardware service, such as AD sampling, such as serial port communication, such as external interrupt trigger, such as the timer service; can also be software services, such as all kinds of communication protocol stack, the fat file system, queue, software filter and so on. A service is typically implemented in one or more functions (a good job division does not allow a service to contain more than 2 or more than one). Simple functions, such as CRC to check the function to get the function to get the results, I would not say; the best use of the task of the best way to achieve the task. When it comes to the task, it will involve the operating system scheduler, or simply a simple state machine. In short, the realization of the task can be understood as a process. Since it is a process, then the task to do is cyclical. For example, the AD sampling task consists of at least 3 steps: channel selection and start sampling, sampling and waiting for sampling, data processing. Such three steps together constitute a task cycle, when the three step is completed, we can think of a cycle is over. To give an example, I2C communications, a complete data packet to send usually contains a series of state constitute a task, when a final state (or some abnormal exit status) ends after, and a task cycle ends.

To sum up, the application of modular, functional tasks, the ultimate goal of the task is the cycle of the final goal is the task cycle. Only if one thing cycles will it have a beginning and an end. Have a beginning and an end, can according to need to issue the "wages", to avoid wasting. And the most common way to do the task is to achieve the most common method of modular service will be independent of the function of the service so as to facilitate management, easy to find the beginning and end of a thing. Find his soldiers, is the starting point for power management. And to do this, it is necessary to have the overall concept of the embedded system, the need for the development of the module based on the development of the service and interface development experience. The accumulation of experience and the global concept is the most complex part.

\section{CONCLUSIONS}

The sleep-awakening technology has applied to a variety of equipment on the transmission line in many electric power companies. In most cases, it has achieved a good energy saving effect. The energy saving effect of this technology is closely associated with the communication mechanism of equipment: the stronger the communications equipment periodically, the better the energy saving effect. But even in completely irregular communication cases, using this technology doesn't make equipment consume more energy.

Once the realization of the task cycle, is equal to the whole system is divided into a number of periodic work tasks, they may seems to be staggered, parallel or no precedence, but essentially said, each small task just care about their initiation and suspension is good. Power management system of the simplification for each task of the power management -- as long as each task does the minimum power consumption, so the whole system in an effective coordination mode, you can do it with minimal power consumption.

According to the above description, the power management based on the task is actually divided into two parts: the power management of the micro angle task and the coordination of the multi task of the macro perspective. 
We start from the microscopic point of view. A task, first of all, certainly can independently accomplish their functions, this seemingly insignificant, is actually very critical, he assured tasks in all the steps are determined, are "themselves", to the outside world "is the black box", in short, is the "autonomy".

On this basis, if required to meet the requirements of low power consumption, nothing more than the following circumstances:

1) task execution process is not allowed to sleep, so task at the beginning and at the end of to set the mark told coordinate system. "As long as I did not say OK is not allowed to sleep" "people in the task in the"

2) task execution process, some phases allows dormant, and also some of the steps is not allowed to sleep; if we will "not allowed to sleep" as a minimum grade of dormancy, then according to the size of the power, dormancy can be from low too high for several grades. In this case, we can modify the above definition as: during the execution of the task, different stages allow different levels of sleep.

3) in the process of the implementation of the task, do not care whether there is a dormancy.

Obviously, if the task also exists in the system, the third task basically is "air" can be ignored, and the first task is quite overbearing, as long as he in execution not allowed sleeping; for the second task, namely, the completion of the task, and take into account the dormancy is a worthy of praise "good comrade". When we design the system, we should try our best to write the two kinds of tasks, and avoid or try to split the first class task.

From a macro point of view, at any moment, there may be more than one task at the same time, so the need for each task is different. How to do it if you want to set up a coordination mechanism? Is this coordination mechanism need to be aware of the details of each task, and then "smart" to find the right time to use the appropriate level to sleep? Too complicated, right? Is actually very simple, let each task sends a representative to the meeting the -- every time the coordinating body to sleep. He called all the delegates voted. Everyone provides a feel able to tolerate the highest dormancy level, the arbiter of the meeting from the vote to find a minimum dormancy level, also is a bucket of the shortest link as the consensus of the meeting ", and then enter the corresponding dormancy level. Obviously, if someone threw a "no dormancy" vote, the arbitration can only choose to give up the dormancy. So, each a "task" should be a responsible task, and not because of their own interests, in order to ensure the execution of their tasks is hasty choice themselves during the period of execution "are not allowed to sleep". The correct approach is that each task should be based on their own different steps in time to update their own tolerance to sleep, so as to ensure the time to meet the meeting, can achieve a meaningful result.

In summary, if each a work are responsible, division of tasks is very reasonable, then through this negotiation mechanism, the system does not exist naturally soldiers who do not pull the horse, can do sleep when needed.

\section{REFERENCES}

[1] CHEN Haibo, WANG Cheng, LI Junfeng, WANG Changfei and XU Guoqing: Application of On-Line Monitoring Technologies for UHV AC Transmission Lines. (Power System Technology 2009, 33(10) : 55-58 In Chinese).

[2] LI Xianzhi, DU Lin, CHEN Weigen, WANG Youyuan, SUN Caixin, LI Jingyan: A Novel Scheme of Draw out Power Supply Utilized in Transmission Line State Monitoring[J] (Automation of Electric Power System 2008, 32(1):76-80 In Chinese)

[3] LIU Hongwei, LI Junfeng, WANG Changfei :Research and Application of On-line Monitoring Management Platform for $1000 \mathrm{kV}$ AC Transmission Lines(Automation of Electric Power System 2009, 33(23) : 98-102 In Chinese).

[4] IEEE 802.16e/D5-2004, Part 16: Air Interface for Fixed and Mobile Broadband Wireless Access Systems Amendment for Physical and Medium Access Control Layers for Combined Fixed and Mobile Operation in Licensed Bands[S].Nov.2004

[5] Yang Xiao Energy Saving Mechanism in the IEEE 802.16e Wireless MAN[J].IEEE Commucications Letters, July 2005,vol.9,No.7,pp.595597

[6] M.Kim,J.Y.Choi,and M.Kang. Performance analysis of the IEEE 802.16e power management for the initiations of awakening[J].The Jounal of China Universities of Posts and Telecom,Oct.2007,vol.14,pp.55-59

[7] N. M. P. Nejatian and M.M. Nayebi. Evaluating the Effect of nonPoisson Traffic Patterns 011 Power Consumption of Sleep Mode in the IEEE802.16e MAC [C]. In: Proceedings of IEEE International Conference on Wireless and Optical Communications Networks, July 2007,pp.1-5

[8] Jung, W.J., Ki, H.J., Lee, T.J., Chung, M.J.: Adaptive sleep mode algorithm in IEEE 802.16e. In: APCC 2007, pp. 483-486

[9] LIU Li, LI JinSheng, HONG PeiLin: Research and Improvement of Sleep Mode in IEEE 802.16e[J].(Chinese Journal Of Computers 2007,vol.30,No.1,pp.146-151 In Chinese) 